\title{
Quantum kinetic equations approach to the description of chemical reactions between adparticles at a metallic surface
}

\author{
V.V.Ignatyuk ${ }^{1}$, M.V.Tokarchuk ${ }^{1}$, P.P.Kostrobij ${ }^{2}$ \\ ${ }^{1}$ Institute for Condensed Matter Physics \\ of the National Academy of Sciences of Ukraine, \\ 1 Svientsitskii Str., 79011 Lviv, Ukraine \\ 2 Lviv Polytechnic National University, \\ Bandery Str.12, Lviv, Ukraine
}

Received August 22, 2005, in final form October 12, 2005

\begin{abstract}
Adparticle dynamics at a metallic surface is studied by means of quantum kinetic equations. Kinetic equations of the reaction-diffusion type are written down for nonequilibrium one-particle distribution functions of the adsorbate up to the second order in the tunnelling constants and amplitudes of chemical reactions.
\end{abstract}

Key words: quantum kinetic theory, models of surface chemical reactions, diffusion coefficient, rate constant

PACS: $05.60 . \mathrm{Gg}, 05.70 . \mathrm{Np}, 63.10 .+\mathrm{a}, 68.43,82.20 . \mathrm{Xr}$

\section{Introduction}

Description of reaction-diffusion processes of particles adsorbed on metallic surfaces is a topical problem of surface physics. Recent investigations in scanning tunnelling microscopy (STM) show [1] that it is possible to visualize direct chemical processes by revealing the fundamental properties of atoms and molecules and their interaction with each other and the environment. A researcher is able to measure the energy transfer, associated with vibrational-rotational particle motion, study diffusion of molecules and their conformational changes, detect bond dissociation or formation in chemical reactions, etc. Though the main purpose of single molecule chemistry experiments is to study the individual molecules, the interactions between adjacent adparticles strongly effect the observed picture, and the individuals start to move cooperatively. Such a phenomenon is observed [2], for instance, during diffusion of hydrogen atoms on $\mathrm{Cu}(001)$. The nature of diffusion changes when two hydrogen atoms are close to each other: the diffusion of a pair rather than of individuals is observed. On the other hand, the reactions of hydrogen transfer $\mathrm{H}_{2} \mathrm{~S}+\mathrm{CC} \longrightarrow \mathrm{HS}+\mathrm{CCH}$ between carbon diatomics and hydrogen sulfide [3,4], involving a number of reacting particles, were observed with high resolution enabling us to trace the bond dissociation/formation and a post-reactional motion of a single molecule.

Therefore, theorists face a new task of creating reliable models that can describe collective surface dynamics of the adparticles with taking into account the inherent features of each molecule. Though the existing theoretical apparatus is capable of describing the whole variety of intra- and intermolecular motions, in fact, such a complex problem is hardly solvable, and a reduction in the level of description becomes indispensable. One of the possible ways is to treat an adsorbed molecule as an unstructured particle that resides on a certain lattice site $^{1}$, at the same time retaining a possibility to introduce all kinds of interactions into the system including chemical reactions.

\footnotetext{
${ }^{1}$ The STM microscopy fairly often detect such a configuration of adparticles location, see [1] and references therein.
}

(C) V.V.Ignatyuk, M.V.Tokarchuk, P.P.Kostrobij 
In this paper we present an approach to the description of a single particle dynamics taking into account a possibility of chemical reactions between adparticles. We treat the chemical transformations as simple bimolecular reactions $\alpha+\beta \longleftrightarrow \alpha^{\prime}+\beta^{\prime}$ between different species of unstructured molecules that reside at a certain end of the lattice cite. The adparticles are capable of performing intrasite oscillations between the ground and first excited states within the quantum well, hop to the nearest lattice site, and interact with lattice vibrations. In section 2 we present a system Hamiltonian, which describes the processes of various nature (intrasite oscillations, tunnelling, adsorbate-substrate interaction, chemical reactions). In section 3 the quantum kinetic equations of the reaction-diffusion type are written down in the second approximation in tunnelling constants and amplitudes of chemical reactions. These equations describe a coherent (non-dissipative) motion of the adsorbate, particle diffusion, and kinetics of chemical reactions. Peculiar to these equations is that both diffusion coefficients and reaction rates are phonon-modified and depend on the lattice parameters (temperature, coupling strengths, mass of a substrate atom etc). In the last section we presented brief conclusions and pointed out some prospects for further investigations.

\section{System Hamiltonian}

To specify all the interactions in the "metallic surface - adparticles" system we choose the Hamiltonian, which permits a site-to-site tunneling of an adsorbate, intrasite oscillation of an adparticle between the ground and excited states within the potential well, interaction of the adparticles with a lattice (a coupling with substrate is both by density and oscillation modes), and bimolecular chemical reactions. We present the Hamiltonian in the following form:

$$
H=H_{\mathrm{A}}+H_{\mathrm{int}}+H_{\text {react }}+H_{\mathrm{B}}
$$

where the fundamental Hamiltonian of the adsorbate is given by a two-band Hubbard Hamiltonian $H_{\mathrm{A}}$ :

$$
\begin{aligned}
H_{\mathrm{A}}= & \sum_{\alpha}\left\{\sum_{\left\langle s s^{\prime}\right\rangle, \sigma}\left(-t_{\alpha 0} a_{\alpha s 0 \sigma}^{\dagger} a_{\alpha s^{\prime} 0 \sigma}+t_{\alpha 1} a_{\alpha s 1 \sigma}^{\dagger} a_{\alpha s^{\prime} 1 \sigma}\right)\right. \\
& \left.+\sum_{s}\left(\frac{\Omega_{\alpha}}{2}\left(n_{\alpha s 1}-n_{\alpha s 0}\right)+U_{\alpha} \frac{n_{\alpha s}\left(n_{\alpha s}-1\right)}{2}\right)\right\}
\end{aligned}
$$

with

$$
n_{\alpha s i}=\sum_{\sigma} n_{\alpha s i \sigma}, \quad n_{\alpha s}=n_{\alpha s 0}+n_{\alpha s 1}
$$

Here the Greek letters denote sort of the particle, $s$ labels the site in the lattice, 0 and 1 are two states (ground and excited) within a given well, $\sigma$ is the spin label, and $\left\langle s s^{\prime}\right\rangle$ denotes a sum over the nearest-neighbour sites. Quantum states within a well are referred to as "vibrational" states, with vibrational frequencies $\Omega_{\alpha} \cdot a_{\alpha s i \sigma}^{\dagger}\left(a_{\alpha s i \sigma}\right)$ creates (destroys) a particle of the $\alpha$-sort on the site $s$, in vibrational state $i$, and with spin $\sigma ; n_{\alpha s i \sigma}=a_{\alpha s i \sigma}^{\dagger} a_{\alpha s i \sigma}$ is the number operator for this state. Depending on the nuclear spin of the adparticles, the creation and destruction operators will obey Bose or Fermi commutation relations; we shall omit the spin label in all subsequent expressions. $t_{\alpha 0}$ and $t_{\alpha 1}$ are the nearest-neighbour tunnelling amplitudes in the ground and first excited states, respectively, and $U_{\alpha}$ means on site Hubbard repulsion between particles.

The coupling to phonons, described by the term $H_{\text {int }}$, is considered to be local within each well. Phonons may couple both to the adsorbate density operators and to the vibrations within a quantum well. The interaction Hamiltonian is

$$
H_{\mathrm{int}}=\sum_{\alpha s}\left\{n_{\alpha s} \sum_{q} \gamma_{\alpha s q}\left(b_{q}+b_{q}^{\dagger}\right)+\left(a_{\alpha s 0}^{\dagger} a_{\alpha s 1}+a_{\alpha s 1}^{\dagger} a_{\alpha s 0}\right) \sum_{q} \chi_{\alpha s q}\left(b_{q}+b_{q}^{\dagger}\right)\right\}
$$


where $b_{q}^{\dagger},\left(b_{q}\right)$ creates (destroys) a phonon with normal mode frequency $\omega_{q}$. Strengths $\gamma_{\alpha s q}\left(\chi_{\alpha s q}\right)$ describe the coupling of phonons to the density (oscillation) modes of the adsorbate and depend on the sort of the particles; for the 1D lattice they could be written explicitly via parameters of the lattice in the following way:

$$
\begin{aligned}
\gamma_{\alpha s q} & =\frac{G_{\alpha}}{\sqrt{M\left(\hbar \omega_{\max }\right)^{3}}} \frac{-2 \hbar \omega_{\max } \sin [\pi /(N+1) q / 2] \cos [\pi /(N+1) q(s+1 / 2)]}{\sqrt{N+1} \sqrt{\omega_{q} / \omega_{\max }}} \\
\chi_{\alpha s q} & =\frac{G_{\alpha}}{\sqrt{M\left(\hbar \omega_{\max }\right)^{3}}} \frac{2 \hbar \omega_{\max } \cos [\pi /(N+1) q / 2] \sin [\pi /(N+1) q(s+1 / 2)]}{\sqrt{N+1} \sqrt{\omega_{q} / \omega_{\max }}} \\
\omega_{q} & =\omega_{\max } \sin [\pi /(N+1) q / 2]
\end{aligned}
$$

where the coupling constant $G_{\alpha}$ depends on the sort of adparticle and could be expressed in terms of matrix elements of the "substrate-adsorbate" interaction potential [5] over the localized Wannier states, $M$ denotes a mass of the substrate atom and $\omega_{\max }$ could be associated with lattice Debye temperature.

The third term in (2.1) is associated with bimolecular chemical reactions $\alpha+\beta \longleftrightarrow \alpha^{\prime}+\beta^{\prime}$ :

$$
H_{\mathrm{ch}}=\sum_{s f, s^{\prime} f^{\prime}} \sum_{i j, i^{\prime} j^{\prime}} \kappa a_{\alpha^{\prime} s^{\prime} i^{\prime}}^{\dagger} a_{\beta^{\prime} f^{\prime} j^{\prime}}^{\dagger} a_{\alpha s i} a_{\beta f j}+\text { h.c. }
$$

where the second (Hermite conjugate) term describes the reverse reaction, $\kappa \equiv\left\langle\alpha^{\prime} s^{\prime} i^{\prime}, \beta^{\prime} f^{\prime} j^{\prime}\left|V_{\text {react }}\right|\right.$ $\alpha s i, \beta f j\rangle$ mean reaction amplitudes, which are assumed to be known from quantum mechanical calculations. The last term in equation (2.1)

$$
H_{\mathrm{B}}=\sum_{q} \hbar \omega_{q} b_{q}^{\dagger} b_{q}
$$

corresponds to the phonon bath.

However, it is useful $[5,6]$ to start from a unitary transformed Hamiltonian on a new correlated basis, which provides a better zeroth-order representation: the sequence of unitary transformations has the effect of changing to a representation in which the adsorbate is localized at one end of an adsorption site or the other, and in which there is a correlated displacement of the lattice. Before performing the unitary transformation, let us pass to the hybrid set of states for each site according to the following rule:

$$
a_{\alpha s_{R}^{L}} \equiv \frac{1}{\sqrt{2}}\left(a_{\alpha s 0} \pm a_{\alpha s 1}\right)
$$

and similarly for the creation operators. The designation $L$ or $R$ means that a single adparticle is now localized on the left or right side of the given well. Then, applying a procedure of double unitary transformation $\tilde{H}=\mathcal{U} H \mathcal{U}^{\dagger}$ with the operator $\mathcal{U}=\mathcal{U}_{1} \mathcal{U}_{2}$, where

$$
\begin{aligned}
& \mathcal{U}_{1}=\prod_{\alpha s} \exp \left[-n_{\alpha s} \sum_{q} \frac{\gamma_{\alpha s q}}{\omega_{q}}\left(b_{q}^{\dagger}-b_{q}\right)\right] \\
& \mathcal{U}_{2}=\prod_{\alpha s} \exp \left[-\left(n_{\alpha s L}-n_{\alpha s R}\right) \sum_{q} \frac{\chi_{\alpha s q}}{\omega_{q}}\left(b_{q}^{\dagger}-b_{q}\right)\right]
\end{aligned}
$$

we obtain a unitary transformed Hamiltonian of the system of chemically reacting adparticles in the following form:

$$
\tilde{H}=\sum_{\alpha s} H_{\alpha s}+\sum_{\alpha} H_{T \alpha}+H_{\mathrm{ch}}+H_{p p}+H_{\mathrm{B}} \equiv H^{\prime}+H_{p p}+H_{\mathrm{B}}
$$


The term $H_{\alpha s}$ describes the lattice-modified intrasite dynamics of the adparticle:

$$
\begin{aligned}
H_{\alpha s} & =\frac{U_{\alpha}}{2} n_{\alpha s}\left(n_{\alpha s}-1\right)-\left(\frac{\Omega_{\alpha}}{2} B_{\alpha s} a_{\alpha s L}^{\dagger} a_{\alpha s R}+\text { h.c. }\right) \\
B_{\alpha s} & =\exp \left[-2 \sum_{q} \frac{\chi_{\alpha s q}}{\hbar \omega_{q}}\left(b_{q}-b_{q}^{\dagger}\right)\right]
\end{aligned}
$$

with lattice induced operator exponent $B_{\alpha s}$; the second term in (2.9)

$$
\begin{aligned}
H_{T \alpha}= & \sum_{\left\langle s s^{\prime}\right\rangle} t_{\mathrm{ch}}^{\alpha}\left(B_{\alpha s s^{\prime}}^{L R} a_{\alpha s L}^{\dagger} a_{\alpha s^{\prime} R}+B_{\alpha s s^{\prime}}^{R L} a_{\alpha s R}^{\dagger} a_{\alpha s^{\prime} L}\right) \\
& +t_{\mathrm{pr}}^{\alpha}\left(B_{\alpha s s^{\prime}}^{L L} a_{\alpha s L}^{\dagger} a_{\alpha s^{\prime} L}+B_{\alpha s s^{\prime}}^{R R} a_{\alpha s R^{\dagger}}^{\dagger} a_{\alpha s^{\prime} R}\right)
\end{aligned}
$$

is the intersite tunnelling term with end-changing (end-preserving) amplitudes $t_{\mathrm{ch}}^{\alpha}\left(t_{\mathrm{pr}}^{\alpha}\right)$, which are the linear combinations $t_{c h}^{\alpha}=1 / 2\left(t_{\alpha 1} \pm t_{\alpha 0}\right)$ of the initial tunnelling amplitudes, while the lattice induced operators $B$ are of the structure

$$
\begin{aligned}
B_{\alpha s s^{\prime}}^{L R, R L} & =\exp \left[-\sum_{q}\left(\Delta_{q}^{\alpha s s^{\prime}} \pm{ }^{(+)} \delta_{q}^{\alpha s s^{\prime}}\right)\left(b_{q}-b_{q}^{\dagger}\right)\right] \\
B_{\alpha s s^{\prime}}^{L L, R R} & =\exp \left[-\sum_{q}\left(\Delta_{q}^{\alpha s s^{\prime}} \pm{ }^{(-)} \delta_{q}^{\alpha s s^{\prime}}\right)\left(b_{q}-b_{q}^{\dagger}\right)\right] \\
\Delta_{q}^{\alpha s s^{\prime}} & =\frac{\gamma_{\alpha s q}-\gamma_{\alpha s^{\prime} q}}{\hbar \omega_{q}}, \quad{ }^{( \pm)} \delta_{q}^{\alpha s s^{\prime}}=\frac{\chi_{\alpha s q} \pm \chi_{\alpha s^{\prime} q}}{\hbar \omega_{q}}
\end{aligned}
$$

The third term in (2.9) is associated with bimolecular chemical reactions $\alpha+\beta \longleftrightarrow \alpha^{\prime}+\beta^{\prime}$, renormalized due to the adparticle-phonons interaction:

$$
H_{\mathrm{ch}}=\sum_{s f, s^{\prime} f^{\prime}} \sum_{i j, i^{\prime} j^{\prime}} \bar{\kappa} B_{\alpha s i, \beta f j}^{\alpha^{\prime} s^{\prime} i^{\prime}, \beta^{\prime} f^{\prime} j^{\prime}} a_{\alpha^{\prime} s^{\prime} i^{\prime}}^{\dagger} a_{\beta^{\prime} f^{\prime} j^{\prime}}^{\dagger} a_{\alpha s i} a_{\beta f j}+\text { h.c. }
$$

where $\bar{\kappa}$ are expressed via linear combinations of the corresponding reaction amplitudes $\kappa$, and the Roman letters $\{i, j\}$ denote $\{L, R\}$-states of the adsorbate. The rate constants become latticemodified as

$$
\begin{aligned}
B_{\alpha s i, \beta f j}^{\alpha^{\prime} s^{\prime} i^{\prime}, \beta^{\prime} f^{\prime} j^{\prime}}= & \exp \left\{-\sum_{q}\left[\left(\gamma_{\alpha^{\prime} s^{\prime} q}+\gamma_{\beta^{\prime} f^{\prime} q}-\gamma_{\alpha s q}+\gamma_{\beta f q}\right)+\left(\delta_{i^{\prime} L}-\delta_{i^{\prime} R}\right) \chi_{\alpha^{\prime} s^{\prime} q}\right.\right. \\
& \left.\left.+\left(\delta_{j^{\prime} L}-\delta_{j^{\prime} R}\right) \chi_{\beta^{\prime} f^{\prime} q}-\left(\delta_{i L}-\delta_{i R}\right) \chi_{\alpha s q}-\left(\delta_{j L}-\delta_{j R}\right) \chi_{\beta f q}\right] \frac{b_{q}-b_{q}^{\dagger}}{\hbar \omega_{q}}\right\} .
\end{aligned}
$$

The fourth term of the Hamiltonian (2.9)

$$
\begin{aligned}
H_{p p}= & \sum_{\substack{\alpha \beta \\
\left\langle s s^{\prime}\right\rangle}}-C_{\alpha s, \beta s^{\prime}}^{D D} n_{\alpha s} n_{\beta s^{\prime}}-2 C_{\alpha s, \beta s^{\prime}}^{D O} n_{\alpha s}\left(n_{\beta s^{\prime} L}-n_{\beta s^{\prime} R}\right) \\
& -C_{\alpha s, \beta s^{\prime}}^{O O}\left(n_{\alpha s L}-n_{\alpha s R}\right)\left(n_{\beta s^{\prime} L}-n_{\beta s^{\prime} R}\right)
\end{aligned}
$$

describes the particle-particle lattice induced interaction with strengths

$$
C_{\alpha s, \beta s^{\prime}}^{D D}=\sum_{q} \frac{\gamma_{\alpha s q} \gamma_{\beta s^{\prime} q}}{\hbar \omega_{q}}, \quad C_{\alpha s, \beta s^{\prime}}^{D O}=\sum_{q} \frac{\gamma_{\alpha s q} \chi_{\beta s^{\prime} q}}{\hbar \omega_{q}}, \quad C_{\alpha s, \beta s^{\prime}}^{O O}=\sum_{q} \frac{\chi_{\alpha s q} \chi_{\beta s^{\prime} q}}{\hbar \omega_{q}}
$$


The $C_{\alpha s, \beta s^{\prime}}^{D D} \equiv C_{\alpha \beta,\left|s-s^{\prime}\right|}^{D D}$ terms correspond to the short-range attractive interaction between adparticles at adjacent sites and, therefore, opposes the Hubbard on-site repulsion. The $C_{\alpha s, \beta s^{\prime}}^{D O} \equiv$ $C_{\alpha \beta,\left|s-s^{\prime}\right|}^{D O}$ terms give a mutual repulsion between particles; the long-range $C_{\alpha s, \beta s^{\prime}}^{O O} \equiv C_{\alpha \beta,\left|s-s^{\prime}\right|}^{O O}$ interactions (determined by the overlap of the lattice distortions associated with each particle) stabilize the system when the particles are on sites $s$ and $s^{\prime}$ and are at the same end of their respective wells.

\section{Kinetic equations for one-particle nonequilibrium functions of adsorbate}

We will consider the first three terms of the Hamiltonian (2.9) as a small perturbation $H^{\prime}$, which allows us to construct a closed system of kinetic equations for one-particle nonequilibrium distribution functions to the second order in $H^{\prime}$. Moreover, the experiments in single molecule chemistry show [1], that the rates of chemical reactions are at least two orders lower than the rates of other dynamic processes, associated with adsorbate (vibration, rotation, diffusion). Hence, we can neglect $\left\langle H_{\mathrm{ch}}\right\rangle_{\mathrm{B}}$ (abbreviation $\langle\cdots\rangle_{\mathrm{B}}$ means averaging over the phonon bath) during derivation of the kinetic kernels.

Using a method of reduced density matrix [7], one can write down the equation for nonequilibrium statistical operator $\rho_{\mathrm{S}}(t)$ of the adsorbate as follows:

$$
\frac{\partial \rho_{\mathrm{S}}(t)}{\partial t}+\frac{1}{\mathrm{i} \hbar}\left[\rho_{\mathrm{S}}(t), H_{\mathrm{S}}\right]=-\frac{1}{\hbar^{2}} \int_{-\infty}^{0} \mathrm{~d} t^{\prime} \exp \left(\epsilon t^{\prime}\right) \operatorname{Tr}_{\mathrm{B}}\left\{\left[H^{\prime},\left[H^{\prime}\left(t^{\prime}\right), \rho_{\mathrm{B}} \rho_{\mathrm{S}}(t)\right]\right]\right\}, \quad \epsilon \rightarrow 0
$$

$H\left(t^{\prime}\right)$ stands for an operator $H^{\prime}$ in the Heisenberg picture

$$
H^{\prime}\left(t^{\prime}\right)=\exp \left[\mathrm{i} t^{\prime} \frac{H_{\mathrm{S}}+H_{\mathrm{B}}}{\hbar}\right] H^{\prime} \exp \left[-\mathrm{i} t^{\prime} \frac{H_{\mathrm{S}}+H_{\mathrm{B}}}{\hbar}\right]
$$

with the Hamiltonian

$$
H_{\mathrm{S}} \equiv H_{p p}^{m f}+\sum_{\alpha s}\left\langle H_{\alpha s}\right\rangle_{\mathrm{B}}+\sum_{\alpha}\left\langle H_{T \alpha}\right\rangle_{\mathrm{B}}=\sum_{\alpha s i} E_{\alpha s i} n_{\alpha s i}+\sum_{\alpha i} \sum_{\left\langle s s^{\prime}\right\rangle} t_{\mathrm{pr}}^{\alpha}\left\langle B_{\alpha s s^{\prime}}^{i i}\right\rangle_{\mathrm{B}} a_{\alpha s i}^{\dagger} a_{\alpha s^{\prime} i} .
$$

In equation (3.19) we have performed a mean field approximation for $H_{p p}$ to obtain a closed form of the kinetic kernels; the mean field "energies" $E_{\alpha s i}$ are the functions of equilibrium adsorbate densities and parameters $C$. This approximation is known to be quite reasonable in the case of low coverage of the adsorbate and short-range nature of the interaction. Taking into account all the approximations mentioned above, multiplying (3.17) by $a_{\alpha c \varkappa}^{\dagger} a_{\alpha c \varkappa}$ and taking the trace over the phase variables of the system, we can write down the kinetic equations for one-particle densities as follows:

$$
\begin{aligned}
\frac{\partial n_{\alpha c \varkappa}(t)}{\partial t}= & \frac{1}{\mathrm{i} \hbar} \sum_{s}\left(\left\langle B_{\alpha c s}^{\varkappa \varkappa}\right\rangle_{\mathrm{B}}\left\langle a_{\alpha c \varkappa}^{\dagger} a_{\alpha s \varkappa}\right\rangle_{\mathrm{S}}^{t}-\text { h.c. }\right)-\frac{1}{\hbar^{2}} \int_{-\infty}^{0} \mathrm{~d} t^{\prime}\left\{\mathcal{K}_{\alpha c \nu}^{\alpha c \nu}\left(t^{\prime}\right) \tilde{\mathcal{K}}_{\alpha s \varkappa}^{\alpha s \varkappa}\left(t^{\prime}\right)\left|V_{\alpha c s}^{\varkappa \nu}\right|^{2} \mathcal{A}\right. \\
& \left.-\mathcal{K}_{\beta f j}^{\beta f^{\prime \prime} j}\left(t^{\prime}\right) \mathcal{K}_{\alpha c \varkappa}^{\alpha s^{\prime \prime} \varkappa}\left(t^{\prime}\right) \tilde{\mathcal{K}}_{\beta^{\prime} f^{\prime} j^{\prime}}^{\beta^{\prime} f^{\prime \prime \prime} j^{\prime}}\left(t^{\prime}\right) \tilde{\mathcal{K}}_{\alpha^{\prime} s^{\prime} i^{\prime}}^{\alpha^{\prime} s^{\prime \prime \prime} i^{\prime}}\left(t^{\prime}\right)|\kappa|^{2} \mathcal{B}\right\}+ \text { c.c. }
\end{aligned}
$$

To obtain a closed system of equations for the one-particle densities we neglect the pair correlation functions during decoupling of the higher order products of the operators $a^{\dagger} a$. This procedure is well-grounded in the second order approximation since the pair correlation functions $g_{2}(t)$ are, at least, of the first order in $H^{\prime}$. In the higher order approximations one can obtain a quantum analog of BBGKY hierarchy for the distribution functions [10], which has to be truncated at a certain level to obtain a closed system of equations.

The first term of the r.h.s. of equation (3.20) with $\operatorname{Sp} \rho_{\mathrm{S}}(t) a_{\alpha c \varkappa}^{\dagger} a_{\alpha s \varkappa}=\left\langle a_{\alpha c \varkappa}^{\dagger} a_{\alpha s \varkappa}\right\rangle_{\mathrm{S}}^{t}$ describes a coherent (nondissipative) motion of the adsorbate and depends on the nonequilibrium tunnelling probabilities; the second term with

$$
\mathcal{A}=\left\langle\Delta B_{\alpha c s}^{\varkappa \nu} \Delta B_{\alpha c s}^{\nu \varkappa}\left(t^{\prime}\right)\right\rangle_{\mathrm{B}}\left(1 \pm n_{\alpha s \nu}(t)\right) n_{\alpha c \varkappa}(t)-\left\langle\Delta B_{\alpha c s}^{\nu \varkappa}\left(t^{\prime}\right) \Delta B_{\alpha c s}^{\varkappa \nu}\right\rangle_{\mathrm{B}} n_{\alpha s \nu}(t)\left(1 \pm n_{\alpha c \varkappa}(t)\right)
$$


is dealt with adparticle diffusion, and the last term with

$$
\begin{aligned}
& \mathcal{B}=\left\langle\Delta B_{\alpha c \varkappa, \beta f j}^{\alpha^{\prime} s^{\prime} i^{\prime}, \beta^{\prime} f^{\prime} j^{\prime}} \Delta\left[B_{\alpha s^{\prime \prime} \varkappa, \beta f^{\prime \prime} j}^{\alpha^{\prime} s^{\prime \prime \prime} i^{\prime}, \beta^{\prime} f^{\prime \prime \prime} j^{\prime}}\left(t^{\prime}\right)\right]^{\dagger}\right\rangle_{\mathrm{B}} n_{\alpha^{\prime} s^{\prime} i^{\prime}}(t) n_{\beta^{\prime} f^{\prime} j^{\prime}}(t)\left(1 \pm n_{\beta f j}(t)\right)\left(1 \pm n_{\alpha c \varkappa}(t)\right) \\
& -\left\langle\left[\Delta B_{\alpha s^{\prime \prime} \varkappa, \beta f^{\prime \prime} j}^{\alpha^{\prime} s^{\prime \prime \prime} i^{\prime}, \beta^{\prime} f^{\prime \prime \prime} j^{\prime}}\left(t^{\prime}\right)\right]^{\dagger} \Delta B_{\alpha c \varkappa, \beta f j}^{\alpha^{\prime} s^{\prime} i^{\prime}, \beta^{\prime} f^{\prime} j^{\prime}}\right\rangle_{\mathrm{B}} n_{\alpha c \varkappa}(t) n_{\beta f j}(t)\left(1 \pm n_{\beta^{\prime} f^{\prime} j^{\prime}}(t)\right)\left(1 \pm n_{\alpha^{\prime} s^{\prime} i^{\prime}}(t)\right)
\end{aligned}
$$

is due to chemical reactions. The upper sign in the kinetic equations corresponds to Bose statistics of the adsorbate, while the lower corresponds to Fermi statistics. Summation over repeated indexes is assumed and restricted to the nearest neighbour (diffusion term) and next-to-nearest neighbour (reaction term) lattice sites. We use the abbreviation $V_{\alpha c s}^{\varkappa \nu}$ to indicate the corresponding intrasite/intresite parameters $\Omega_{\alpha}, t_{\mathrm{ch}}^{\alpha}, t_{\mathrm{pr}}^{\alpha}$.

Equation (3.20) has a typical reaction-diffusion structure; the integral terms in the second order approximation depend only on $n_{\alpha s i}(t)$, and there is no cross-correlation between processes of different nature (intrasite/intersite motion, chemical reactions). However, it could be shown that in the higher order approximations one can obtain cross-correlation terms. Kinetic equations for tunnelling probabilities $\left\langle a_{\alpha c \varkappa}^{\dagger} a_{\alpha s \varkappa}\right\rangle_{\mathrm{S}}^{t}$ are of a similar structure to (3.20) and are not presented here explicitly for the sake of brevity. A remarkable feature of the system of kinetic equations (3.20) is that the integral terms in the second order approximation depend only on $n_{\alpha s i}(t)$ and the only coupling between nonequilibrium densities and nonequilibrium tunnelling probabilities are due to the coherent terms.

Kinetic kernels, formed by particle dynamics, are expressed via Bessel functions $J$, mean field "energies" $E$ (which are the functions of equilibrium adsorbate coverage and parameters $C$ ), and renormalized tunnelling amplitude $\tau_{s s^{\prime} \varkappa}^{\alpha}=t_{\mathrm{pr}}^{\alpha}\left\langle B_{\alpha s s^{\prime}}^{\varkappa \varkappa}\right\rangle_{\mathrm{B}}$ as follows:

$$
\begin{aligned}
\mathcal{K}_{\alpha s^{\prime} \varkappa}^{\alpha s}\left(t^{\prime}\right) \tilde{\mathcal{K}}_{\beta f^{\prime} j}^{\beta f}\left(t^{\prime}\right)= & \mathrm{i}^{|s-f|+\left|s^{\prime}-f^{\prime}\right|} J_{\left|s-s^{\prime}\right|}^{D}\left(2 \tau_{s s^{\prime} \varkappa}^{\alpha} t^{\prime}\right) J_{\left|f-f^{\prime}\right|}^{D}\left(2 \tau_{f f^{\prime} j^{\prime}}^{\beta} t^{\prime}\right) \\
& \times \exp \left[\frac{\mathrm{i}}{\hbar}\left(E_{\alpha s \varkappa}-E_{\beta f j}\right) t^{\prime}\right] .
\end{aligned}
$$

Hereafter $D$ denotes the lattice dimensionality.

An explicit expression for $\left\langle B_{\alpha s s^{\prime}}^{\varkappa \mathrm{B}}\right.$ could be written down as a square root of the second term of equation (3.22). It follows from the analysis of spectral functions in case of the infinite lattice $[5,6]$ as well as from the expressions (2.4) for the finite lattice that the end-changing renormalizing factors $B$ tend to zero after the averaging over a thermal bath. It is seen from equation (3.21), that the main contributions to the kinetic kernels are due to the zeroth order Bessel functions; the same analysis yields that the values of nonequilibrium tunnelling probabilities are proportional to the Bessel functions of higher orders and, hence, turn out to be small ${ }^{2}$. It should be stressed that kinetic kernels (3.21) decay in time due to the tunnelling processes. Thus, both generalized diffusion coefficients and generalized rates of chemical reactions are finite. Another way of obtaining finite diffusion coefficients is to take into consideration an interaction between adparticles and electrons of a substrate [8], but obviously this problem is much more complicated.

The kinetic kernels formed by the lattice dynamics

$$
\left\langle\Delta B_{I} \Delta B_{I}\left(t^{\prime}\right)\right\rangle_{\mathrm{B}}=\exp \left[-\left(\varphi_{I}(0)-\varphi_{I}\left(t^{\prime}\right)\right)\right]-\exp \left[-\varphi_{I}(0)\right]
$$

depend on the lattice parameters (coupling strengths, temperature, mass of the lattice atoms) via

$$
\varphi_{I}\left(t^{\prime}\right)=\sum_{q} \Gamma_{I}(q)^{2}\left(\operatorname{coth}\left[\hbar \omega_{q} / k_{\mathrm{B}} T\right] \cos \left(\omega_{q} t^{\prime}\right)-\mathrm{i} \sin \left(\omega_{q} t^{\prime}\right)\right),
$$

where $\Gamma_{I}(q)$ denote the corresponding combinations of the lattice parameters $\gamma_{\alpha s i}$, $\chi_{\alpha s i}$ in the phonon induced exponents (2.10), (2.12), (2.14).

\footnotetext{
${ }^{2}$ The main contribution to the kernels (3.21) originates from the functions, which are finite at $t^{\prime}=0$ (zeroth order $J_{\mathrm{s}}$ ) in contrast to higher order Bessel functions, which are zero at $t^{\prime}=0$.
} 
It is seen from the last equation (2.4) that in the case of infinite lattice $(N \rightarrow \infty)$ there is no gap in the spectrum of lattice vibration $\omega_{q}$, and one has to describe the adsorbate-substrate interactions by means of spectral weight functions $\mathcal{J}_{\alpha,\left|s-s^{\prime}\right|}^{\mathrm{ch} / \mathrm{pr}}(\omega)$. The main contributions to the static and dynamic correlation functions originate from the low frequency domain of the $\mathcal{J}_{\alpha,\left|s-s^{\prime}\right|}^{\mathrm{ch} / \mathrm{pr}}(\omega)$. In statics, different behaviour of end-changing spectral functions $\left(\mathcal{J}_{\alpha,\left|s-s^{\prime}\right|}^{c h}(\omega) \sim \omega^{D-2}\right.$ at low $\left.\omega\right)$ and end-preserving spectral functions $\left(\mathcal{J}_{\alpha,\left|s-s^{\prime}\right|}^{p r}(\omega) \sim \omega^{D}\right.$ at low $\left.\omega\right)$ results in self-trapping of the adsorbate in the certain end of the potential well due to the overlap of lattice distortions and renormalization of phonon induced tunnelling amplitudes (2.12) and intrasite oscillation frequency $\Omega_{\alpha} B_{\alpha s}$. In dynamics, the same factor leads to different activation behaviour for the diffusion constants [6] and chemical rates, arising from the form of correlation functions in the kinetic kernels.

Thus, in the case of the infinite lattice one can rewrite the equation (3.23) via spectral functions as follows:

$$
\varphi_{I}\left(t^{\prime}\right)=\int_{0}^{\omega_{\max }} \frac{\mathcal{J}_{I}(\omega)}{\omega^{2}}\left(\operatorname{coth}\left[\hbar \omega / k_{\mathrm{B}} T\right] \cos \left(\omega t^{\prime}\right)-\mathrm{i} \sin \left(\omega t^{\prime}\right)\right) \mathrm{d} \omega
$$

where, for instance, the intrasite spectral function could be written as

$$
\mathcal{J}_{\alpha s}(\omega)=\sum_{q=1}^{\infty} \chi_{\alpha s q}^{2} \delta\left(\omega-\omega_{q}\right) \sim \omega^{D-2}
$$

and frequency characteristics for the other spectral functions (with $I$ corresponding to the tunnelling processes and chemical reactions) could be obtained after some algebra.

To complete the analysis of kinetic equations let us conclude, that one can obtain temperature dependent diffusion constants and reaction rates with activation energies that are functions of a whole set of the lattice parameters and tunnelling amplitudes. Depending on the underlying processes this could be done by separating the corresponding time scales ${ }^{3}$ before integrating over $t^{\prime}$ in the kinetic kernels or by a series expansion of equation $(3.22)$ in $\varphi_{I}(t)$ (that corresponds to the one- and multiphonon processes being taken into account) in the way it was performed in [6]. In the single particle limit $(N \rightarrow 1)$ and in the strong coupling case $\left(G_{\alpha}\right.$ is comparable with $\left.k_{\mathrm{B}} T\right)$, neglecting off-diagonal elements in the kernels of the kinetic equations (3.20), we obtain for diffusion constant $\mathcal{D}$ :

$$
\mathcal{D} \sim \frac{\exp \left(-E_{a} / k_{\mathrm{B}} T\right)}{\sqrt{k_{\mathrm{B}} T}}
$$

with activation energy $E_{a}$ depending on $G_{\alpha}$. In the weak coupling limit $\left(G_{\alpha} / k_{\mathrm{B}} T \ll 1\right)$ the temperature behaviour of $\mathcal{D}$ is much more complicated (see section 5 of [6]) and cannot be described via a single activation energy. It would be challenging to investigate the temperature dependance of the rate constants since the problem seems to be much more difficult due to the interplay between different $G_{\alpha}$ and, hence, multitude of spectral characteristics depending on the sort of the reacting particles. Changing the strength of coupling between adparticles and a substrate before and after the reaction, it is possible to model the activation behaviour of the rate constants and, consequently, even to manipulate some reaction channels. Thus, a feedback in the complex "theoryexperiment" could be established: on the one hand, it is possible to foresee the peculiarities in the cooperative dynamics of chemically reacting adparticles, and, on the other hand, to visualize the direct chemical processes by revealing the fundamental properties of atoms (molecules) and their interaction with each other and the environment that could supply the theorists with necessary input parameters for the system modelling.

\footnotetext{
${ }^{3}$ Time decay of the kernels (3.21), formed by particle dynamics, is much slower than the characteristic evolution times of the kernels (3.22), formed by the lattice dynamics.
} 


\section{Conclusions}

In the previous sections we presented a quantum-kinetic approach to the description of chemical reactions between unstructured particles adsorbed on the metallic surface and interacting with substrate vibrations. We obtained a system of coupled equations for one-particle nonequilibrium distribution functions and nonequilibrium tunnelling probabilities. These equations are of a reaction-diffusion type and have phonon modified diffusion coefficients and reaction rates, which depend on the lattice parameters. By integrating the kinetic kernels over $t^{\prime}$ it is possible to obtain a temperature dependance of the mentioned characteristics and calculate their activation energies.

However, as we have already mentioned, the observed picture of chemical reactions on the metallic surfaces is much more complicated than the model introduced in the section 2 . The reactants consist of a number of constituent atoms, possess the internal degrees of freedom (stretch/bending vibrations, rotations, conformational changes), are subjected to chemical bond formation/dissociation. Besides, a new class of interactions could be introduced in the system, for instance - the interaction between adparticles and electrons of a substrate. Eventually, one can consider a phonon subsystem to be nonequilibrium that opens a straight road to the description of an interesting phenomenon such as surface reconstruction $[9,10]$. Thus, to conclude the paper we would like to summarize some open questions and problems still to be solved:

- generalization of our ( $\mathrm{L}-\mathrm{R})$ model to the single band and many band cases; this is straightforward;

- calculation of the activation energies for the diffusion coefficients and rate constants; it could be done explicitly in some cases. Alternatively, numerical evaluations could be performed;

- derivation of reaction-diffusion equations in the higher approximations in tunnelling amplitudes and reaction constants;

- taking into account the interactions between the adsorbed particles and the electrons of a substrate; this problem, though being complicated, can be solved by methods of quantum kinetics [8].

- consideration of the bound states of molecules; the existing quantum mechanical apparatus makes it possible to do this (see, for instance, [11]).

In the general case, nonequilibrium distribution functions and tunnelling probabilities could be calculated only numerically for the lattice of a definite structure and the parameters taken from the experiments.

\section{Acknowledgement}

This work was partially supported by Project "Mathematic modelling of the catalytic processes in metallic nanostructures" (Lviv Polytechnic National University, No. 2201020). 


\title{
References
}

1. Ho W., Journ. Chem. Phys., 2002, 117, 11033-11061.

2. Lauhon L.J., Ho W., Phys. Rev. Lett., 2000, 85, 4566.

3. Lauhon L.J., Ho W., Faraday Discuss., 2000, 117, 249.

4. Lauhon L.J., Ho W., Journ. Phys. Chem, 2000, 105, 3987.

5. Reilly P.D., Harris R.A., Whaley K.B., Journ. Chem. Phys., 1991, 95, 8599-8615.

6. Reilly P.D., Harris R.A., Whaley K.B., Journ. Chem. Phys., 1992, 97, 6975-6990.

7. Zubarev D.N., Morozov V.G., Röpke G. Statistical Mechanics of Nonequilibrium Processes. Vol. 2, Relaxation and Hydrodynamic Processes. Akademie Verlag, Berlin, 1996.

8. Zubarev D.N., Morozov V.G., Röpke G. Statistical Mechanics of Nonequilibrium Processes. Vol. 1. Fizmatlit, Moscow, 2002 (in Russian).

9. Riffe D.M., Wertheim G.K., Citrin P.H., Phys. Rev. Lett., 1990, 65, 219.

10. Kostrobii P.P., Rudavskii Yu.K., Ignatyuk V.V., Tokarchuk M.V., Cond. Matt. Phys., 2003, 4, No. 3(35), 409-424.

11. Peletminskii S.V., Slyusarenko Yu.V., Journ. Math. Phys., 2005, 46, 022301.

\section{Квантово-кінетичний підхід до опису хімічних реакцій між частинками адсорбату на металічній поверхні}

\author{
В.В.Ігнатюк ${ }^{1}$, М.В.Токарчук ${ }^{1}$, П.П.Костробій² \\ 1 Інститут Фізики конденсованих систем НАН України, \\ 79011 Львів, вул. Свєнціцького, 1 \\ 2 Національний Університет “Львівська Політехніка”, \\ 79000 Львів, вул. Ст.Бандери, 12 \\ Отримано 22 серпня 2005 р., в остаточному вигляді - \\ 12 жовтня 2005 p.
}

Динаміка частинок, адсорбованих на поверхні металу, досліджується методом квантової кінетики. Отримано систему реакційно-дифузійних кінетичних рівнянь для одночастинкових нерівноважних функцій розподілу адсорбату в другому порядку за константами тунелювання та амплітудами хімічних реакцій.

Ключові слова: квантово-кінетична теорія, моделі хімічних реакцій на поверхні, коефіцієнти дифузії, швидкості реакцій

PACS: $05.60 . \mathrm{Gg}, 05.70 . \mathrm{Np}, 63.10 .+a, 68.43,82.20 . \mathrm{Xr}$ 
